\title{
Metástasis orbitaria e intracraneal secundaria a carcinoma de tiroides
}

\author{
Orbital and intracranial metastasis secondary to thyroid carcinoma
}

José F. Sánchez-Sánchez ${ }^{1 *}$, Yazmin Lemus-Rodríguez, Ricardo Ramírez-Aguilar², Andrés A. Briseño-Hernández y Marcela Ramos-Barrales ${ }^{3}$

${ }^{1}$ Departamento de Neurocirugía, Hospital Regional Dr. Valentín Gómez Farías, Instituto de Seguridad y Servicios Sociales de los Trabajadores del Estado (ISSSTE), Zapopan, Jalisco; ${ }^{2}$ Departamento de Neurocirugía, Hospital General Balbuena, Ciudad de México; ${ }^{3}$ Departamento de Anatomía Patológica, Hospital Regional Dr. Valentín Gómez Farías, ISSSTE, Zapopan, Jalisco; ${ }^{4}$ Departamento de Rehabilitación, Torre Médica Árbol de la Vida, Metepec, Estado de México. México

\section{Resumen}

Introducción: En México existe un aumento exponencial del cáncer de tiroides, con 12,122 nuevos casos en 2018, representando el cáncer endocrinológico más frecuente descrito por la Organización Mundial de la Salud. Se le atribuyen lesiones metastásicas locales y a distancia, siendo una presentación inusual la afección orbitaria. Caso clínico: Mujer de 56 años diagnosticada de leucemia mieloide crónica hace 19 años, que acude por cefalea, proptosis e hiperemia conjuntival con limitación a la abducción, y dolor facial izquierdo. Conclusiones: En estos casos, el tratamiento quirúrgico es indispensable para mejorar la sintomatología y la calidad de vida del paciente.

Palabras clave: Metástasis orbitaria. Proptosis. Cáncer de tiroides. Carcinoma papilar de tiroides. Tumor maligno orbitario.

\begin{abstract}
Introduction: An exponential increase in thyroid cancer has been reported in Mexico with 12,122 new cases in 2018 it represents the most common endocrinological cancer described by the World Health Organization. Attributing it local and distant metastatic lesions, being unusual the orbital condition Clinical case: A 56-year-old woman diagnosed with chronic myeloid leukemia 19 years ago, presented with left eye proptosis and tearing, headaches, worsening left facial neuralgia. Conclusions: In these cases, the surgical treatment is essential to improve patient's symptoms and quality of life.
\end{abstract}

Key words: Orbital metastasis. Proptosis. Thyroid cancer. Papillary thyroid carcinoma. Orbital malignant tumor.

\footnotetext{
Correspondencia:

*José F. Sánchez-Sánchez

Avda. Soledad Orozco 203

El Capullo

Fecha de recepción: 22-11-2020

C.P. 45100 Zapopan, Jal., México

E-mail: jfsanchezsnz@gmail.com

Cir Cir. 2021;89(S1):76-81

Contents available at PubMed www.cirugiaycirujanos.com 0009-7411/@ 2021 Academia Mexicana de Cirugía. Publicado por Permanyer. Este es un artículo open access bajo la licencia CC BY-NC-ND (http://creativecommons.org/licenses/by-nc-nd/4.0/).
} 


\section{Introducción}

En la población mexicana se ha incrementado el número de pacientes con metástasis a distancia, detectándolas en un $60 \%$ en etapas avanzadas. El cáncer de tiroides ocupa el cuarto lugar en incidencia de cáncer en México, siendo el segundo cáncer más frecuente del sexo femenino, desplazando al cáncer cervicouterino a la tercera posición ${ }^{1}$. El carcinoma diferenciado de tiroides, el cual incluye el carcinoma papilar y folicular, es la variante histológica más frecuente de metástasis a distancia, y afecta principalmente el pulmón, el hueso, el hígado y el cerebro; la afección orbitaria es poco frecuente y representa solo el $3 \%$ de la patología orbitaria ${ }^{2}$.

\section{Caso clínico}

Mujer de 56 años con antecedentes de asma y diagnóstico en otra unidad hospitalaria de leucemia mieloide crónica hace 19 años, tratada a base de quimioterapia, interferón alfa y descompresión orbitaria con colocación de metilmetacrilato por lesión única metastásica. Durante su seguimiento en consulta externa continuó con incremento de la proptosis debido a crecimiento tumoral crónico, siendo referida a nuestra unidad por aumento de la sintomatología, predominando la cefalea y dolor tipo neurálgico que no respondía a medicamentos, acompañados de exoftalmos, epífora y alteraciones motoras del globo ocular. En su valoración se observa un aumento de volumen en la región anterior del cuello. Durante la exploración física se encontraron pupilas isocóricas reactivas de $3 \mathrm{~mm}$ y la fundoscopia izquierda mostró una papila con bordes nítidos, de color blanco nacarado, sugestivo de atrofia óptica, proptosis izquierda e hiperemia conjuntival, limitación al cierre palpebral y a los movimientos oculares con afección en la abducción, así como disestesia en territorios de V1 y V2 de la hemicara homolateral (Fig. 1). A la palpación del cuello se encuentra un aumento de volumen de la glándula tiroides acompañado de ganglios no dolorosos de predominio izquierdo. Se realizaron perfiles tumorales y endocrinológicos, presentando solo un aumento de la tirotropina de $7.1 \mathrm{mUI} / \mathrm{l}$. La resonancia magnética de cráneo con gadolinio mostró una lesión única sólida intraconal que se extendía por el borde lateral de la órbita y el ápex, con invasión de los segmentos intracraneal e intracanalicular del nervio óptico y desplazamiento del segmento intraorbitario con extensión al seno cavernoso homolateral (Fig. 2). En su primera intervención se realizó un abordaje orbitocigomático, por lo cual se realizó reapertura del mismo; al retirar el material de reconstrucción con metilmetacrilato de la órbita se encuentran preservados el techo y la pared lateral orbitaria, por lo que se decide extender la orbitotomía. Previo a la apertura de la periórbita se procedió a realizar una clinoidectomía anterior extradural para descomprimir el foramen óptico, así como peeling de la fosa temporal y apertura del foramen supraorbitario para descompresión del ramo V1 del nervio trigémino. Durante la resección tumoral se observó una lesión blanquecina pétrea con infiltración del músculo recto lateral y superior, así como del nervio óptico en su segmento intracanalicular, por lo que se decidió realizar citorreducción con aspirador ultrasónico para disminuir el riesgo de morbilidad en comparación con una resección total (Fig. 3). El estudio histopatológico reportó presencia de tejido fibroconectivo con lesión epitelial infiltrante con distribución en papilas y células poligonales, compatible con lesión metastásica de tiroides. Se realizó biopsia por aspiración con aguja fina (BAAF) de nódulo tiroideo bajo sospecha de cáncer primario, y se encontró una lesión sólida clasificada como Bethesda $\mathrm{V}$ con riesgo de malignidad del 60-75\%. (Fig. 4). En el posoperatorio inmediato se presentó anisocoria izquierda por manipulación del nervio oculomotor, resuelta al día 5 posquirúrgico con mejoría de la sintomatología facial y orbitaria. Durante su seguimiento en la consulta externa se observa una disminución importante en el grado de proptosis, resolviéndose la hiperemia conjuntival y con mayor capacidad en el cierre palpebral (Fig. 5).

\section{Discusión}

El cáncer de tiroides ha tenido un incremento importante en la población mexicana, obteniendo el cuarto lugar respecto a la incidencia de cáncer en México, siendo el $6.4 \%$ del total de nuevos casos en 1 año'. Como ya se mencionó, el cáncer de tiroides se relaciona con una diseminación local y posteriormente a distancia. La variante papilar es la causa más común de extensión local, con infiltración a los tejidos adyacentes, con predominio en los ganglios linfáticos incluyendo la laringe y el esófago; en comparación la variante folicular, causa un mayor número de metástasis a distancia, que aparecen en el siguiente orden de frecuencia: pulmón, hueso, hígado y cerebro ${ }^{2,3}$. En Europa, los sitios de metástasis 


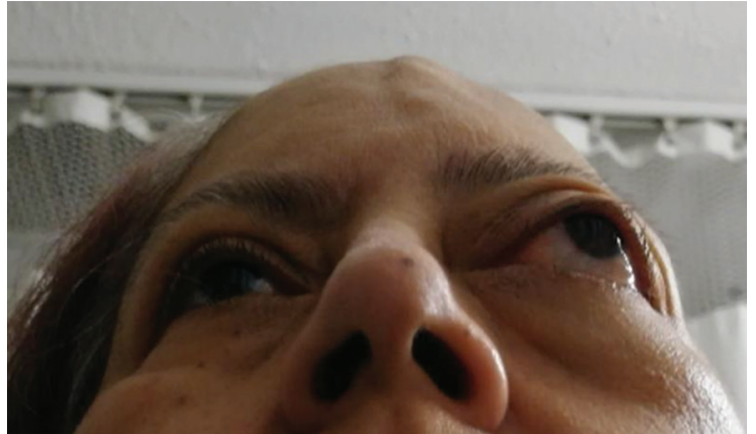

Figura 1. Manifestaciones clínicas: oftalmoplejía y proptosis izquierda.

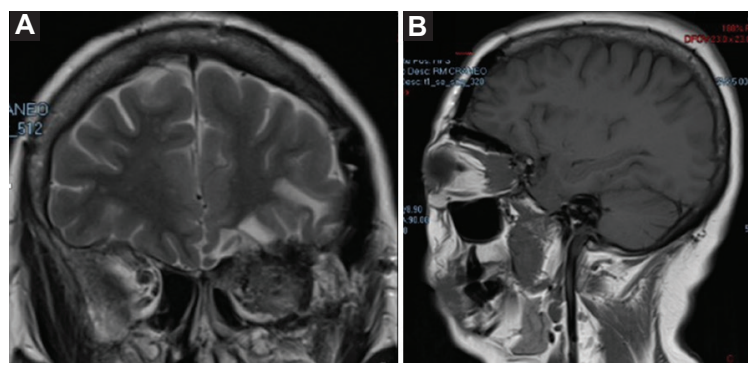

Figura 2. Estudios de imagen. A: resonancia magnética (RM) de cráneo en secuencia T2, corte coronal, que muestra una lesión única hipertensa con invasión del techo, la pared lateral y la grasa periorbitaria. B: RM de cráneo en secuencia T1 que muestra una lesión intraconal que produce efecto de masa con engrosamiento de los músculos extraoculares.
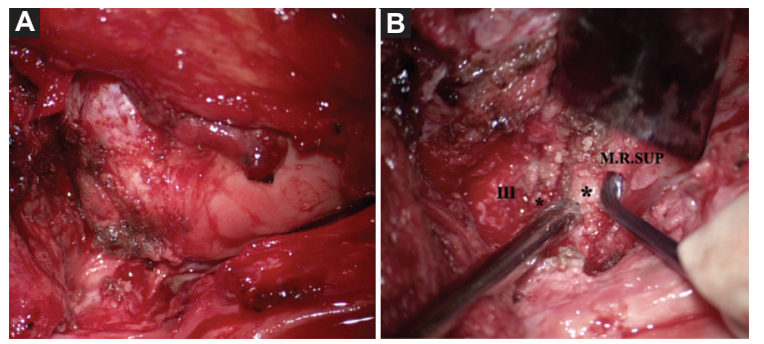

Figura 3. A: abordaje orbitocigomático con peeling de la fosa temporal y apertura de la fisura orbitaria con clinoidectomía anterior extradural. B: apertura y retracción de la periórbita con apoyo de disector, observando infiltrado tumoral sobre el músculo recto superior (M.R.SUP) y el nervio oculomotor (III).

a distancia más frecuentes son el pulmón (56.1\%), el hueso $(28.1 \%)$, la piel $(5.3 \%)$ y el cerebro $(3.5 \%)^{4}$, con una incidencia similar a la de nuestro país, siendo raras también las metástasis a la región orbitaria. Recordando la fisiopatología de las metástasis, las células tumorales se extravasan y migran a través del estroma tisular a un sitio secundario de crecimiento, donde desarrollan un fenotipo molecular

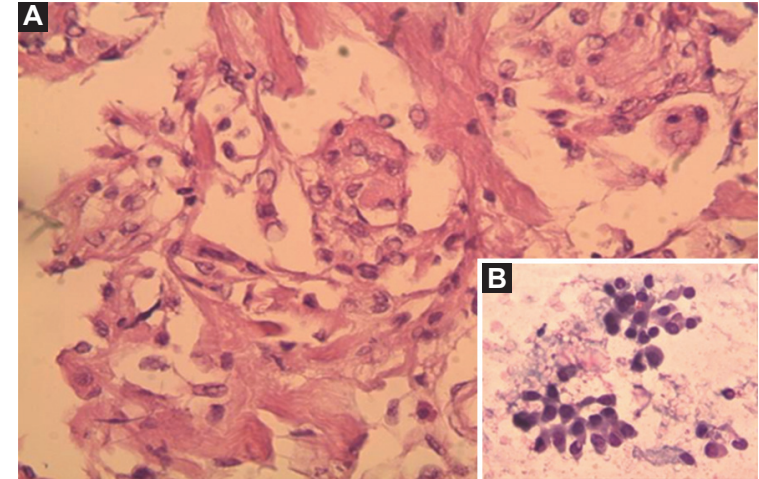

Figura 4. Tinción de hematoxilina-eosina. A: biopsia de tumor orbitario. Distribución en papilas ramificadas con un tallo fibrovascular, mostrando células poligonales con núcleos con cromatina dispersa en «vidrio deslustrado». B: biopsia por aspiración con aguja fina de tiroides. Se observa un acúmulo de células epiteliales de núcleos hipercromáticos con indentaciones nucleares.
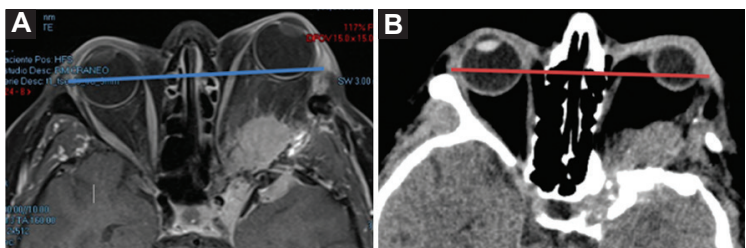

Figura 5. Estudios de imagen. A: resonancia magnética de cráneo con gadolinio, secuencia T1, corte axial, que muestra una lesión única intraconal $(41 \times 18 \times 17 \mathrm{~mm}$ en sus ejes anteroposterior, transversal y longitudinal), que se refuerza con el medio de contraste, mostrando invasión de la pared lateral y el ápex orbitario que involucra la grasa intraconal y extraconal provocando un desplazamiento de los segmentos intraorbitario, intracanalicular e intracraneal del nervio óptico, con extensión al seno cavernoso homolateral. B: tomografía computada de cráneo contrastada posoperatoria, corte axial, en la que se observa la reducción de la proptosis izquierda.

metastásico y un desequilibrio citoesquelético para favorecer la angiogénesis mediante una alteración en la adhesión de proteínas de superficie y la interacción estroma-tumor, estableciendo un adecuado entorno para aumentar el potencial metastásico ${ }^{5}$. De esta manera, la metástasis, tanto local como a distancia, compromete la función de los sitios de invasión, ya sea por compresión, destrucción local o inhibición de su función ${ }^{5}$. El drenaje linfático de la región orbitaria es una vía de diseminación metastásica. El drenaje anterior recoge la linfa que se dirige a los ganglios linfáticos submaxilares, parotídeos y preauriculares, mientras que el drenaje posterior se dirige a las cadenas ganglionares y yugular externa ${ }^{5,6}$. Otra vía de propagación es la hematógena, incluyendo la diseminación por medio del flujo 
venoso del plexo vertebrobasilar ${ }^{5,6}$. Estos tumores metastásicos constituyen aproximadamente el 3\% de todas las patologías de la región orbitaria, representando solo el $2-10 \%$ de las neoplasias de esta región ${ }^{5,6}$. En una exhaustiva revisión de la literatura se mencionan diferentes porcentajes de aparición, dependiendo del grupo étnico y de la edad. Se ha observado que en la infancia el origen de las metástasis orbitarias más frecuente es el linfoma no Hodgkin, seguido de la leucemia (predominando en las formas crónicas) $)^{6,7}$. En la serie de 1264 pacientes de Shields, et al. ${ }^{8}$, en la cual estudiaron las múltiples patologías orbitarias, hubo 91 pacientes $(7 \%)$ con patología metastásica, cuyo origen primario más frecuente fue el cáncer de mama $(48 \%)$, seguido del cáncer prostático $(12 \%)$, melanoma (12\%) y el cáncer de pulmón (8\%). En la serie del departamento de oftalmología de la Universidad de Nagasaki ${ }^{5}$, la causa más frecuente fue el adenocarcinoma de pulmón, con una aparición en hombres cuatro veces mayor que en mujeres, seguido de las neoplasias de mama, hígado y glándula suprarrenal. Las series europeas muestran como sitio primario la mama (39\%), el riñón (11\%), el pulmón (9\%) y la piel $(6 \%)$; sin embargo, el $17 \%$ de los casos tienen etiología indeterminada, colocando en el noveno lugar a la glándula tiroides como origen primario con solo un $1 \%$ de frecuencia 9 . La relación de metástasis a distancia del carcinoma de tiroides al cráneo involucra predominantemente la línea media, siendo el primer lugar de afección el hueso occipital y parietal, y por último la región frontal ${ }^{10}$, observando lesiones intracraneales en el clivus, la hipófisis y la silla turca, y siendo más frecuente la invasión en el seno esfenoidal y el seno cavernoso $0^{10-12}$. Goldberg, et al. ${ }^{13}$ mostraron las frecuencias de afectación de las paredes orbitarias, y establecieron como sitios más comúnmente afectados, en orden de frecuencia, la pared lateral o externa (39\%), el techo $(32 \%)$, la pared medial o interna (20\%), y el piso (12\%); en cuanto al porcentaje de afección de tejidos y porciones de la órbita, el hueso es el sitio más afectado, hasta en un $39 \%$ de los casos, seguido de la grasa periorbitaria $(39 \%)$, el músculo $(18 \%)$ y patrón difuso $(4 \%)^{13,14}$. Entre los componentes musculares mayormente afectados se encuentran, en orden de frecuencia, los músculos rectos horizontales (recto lateral y recto medial), los músculos rectos verticales (recto superior e inferior) y los músculos oblicuos $^{13,14}$. La clínica es similar en las diferentes series de casos, basándonos en la clasificación de
Goldberg que establece cinco presentaciones de lesión orbitaria caracterizadas por ciertos signos y síntomas, siendo el efecto de masa palpable la presentación más frecuente, en el $66 \%$ de los casos, seguido de la presentación infiltrativa (24\%) caracterizada por diplopía y limitación de los movimientos oculares, funcional (5\%) establecida por alteración sensitiva y motora de nervios craneales (II-VI), inflamatoria $(5 \%)$ representada por quemosis y dolor al movimiento ocular, y silente $(<1 \%)^{13}$. Es común que estos síndromes no sean puros y se observe una clínica variable; incluso hasta el $20 \%$ de los pacientes cursan con parestesias o disestesias faciales debidas a la expansión tumoral y el efecto de compresión $^{14,15}$. Los carcinomas tiroideos, gástricos, linfomas y sarcomas producen lesiones osteolíticas de la órbita, sugiriendo un tumor hipervascularizado, acompañadas de dolor ocular con percepción de pulsatilidad debida a la osteólisis ${ }^{5,16,17}$. Es necesario estudiar a los pacientes que presenten proptosis como primer signo de una patología neoplásica hasta demostrar lo contrario; cabe aclarar que no es la primera etiología por considerar, y durante el interrogatorio se deben descartar patologías inflamatorias (oftalmopatía de Graves) e infecciosas (celulitis periorbitaria) ${ }^{16,17}$. El examen clínico deberá incluir un test de medición de la agudeza visual y una exploración de fondo de ojo, pudiéndose observar un defecto pupilar aferente y estrías coroideas por invasión tumoral y disminución en la agudeza visual secundaria a la compresión o la elongación del nervio óptico ${ }^{16,17}$. El aumento del volumen de la glándula tiroides obliga a realizar estudios de laboratorio e imagen que puedan descartar patología tumoral. Se deberá solicitar la determinación de los valores de tiroglobulina en suero, incluso en los pacientes operados de carcinoma tiroideo, ya que unas cifras elevadas indican enfermedad metastásica o recidiva, siendo el principal valor predictivo en el seguimiento de estos pacientes ${ }^{16-18}$. Las guías de la American Thyroid Association señalan a la ecografía como el estudio idóneo para el diagnóstico y la estadificación del riesgo de malignidad. En los pacientes con alta sospecha de neoplasia será necesario realizar una BAAF para corroborar el diagnóstico y la variante ${ }^{18}$. La inmunohistoquímica resulta útil, sobre todo en combinación con tinciones, para el diagnóstico diferencial de las variantes papilar y folicular, mostrando la tinción HBME-1 una sensibilidad del $79-87 \%$ y una especificidad del $83-96 \%$, mientras que la citoqueratina 19 presenta una positividad del 
$86.2 \%$ en los carcinomas papilares ${ }^{18,19}$. Los factores importantes como predictores de mortalidad y gravedad del padecimiento incluyen la edad, la extensión de la invasión y el tamaño tumoral, pero el más importante es la histología. Entre los estudios de imagen iniciales se encuentra la ecografía Doppler ocular y orbitaria, que identifica una masa con características sólida y vascular, demostrando el aumento de tamaño de los músculos extraoculares ${ }^{18,19}$. Ante la sospecha de lesiones malignas será necesario complementar con estudios de imagen especializados, como la tomografía computada tridimensional, que proporciona detalles anatómicos incluyendo la extensión de la invasión tumoral al hueso, sobre todo en casos de osteólisis orbitaria, y medición del defecto que pudiera encontrarse posterior a la craniectomía, permitiendo la planeación de una plastia. La resonancia magnética de órbita y cerebral define el grado de extensión del tumor y las estructuras vecinas afectadas, y permite establecer el mejor abordaje para la resección. Se han realizado numerosos estudios de cohorte retrospectivos basados en la realización únicamente de tratamiento quirúrgico en comparación con resección más radioterapia posoperatoria, y se ha observado un aumento de la supervivencia de hasta 12 meses más que la media con los tratamientos combinados; esto en metástasis orbitaria y craneal única, sin lesiones extracraneales ${ }^{19,20}$. El uso de radioterapia es una buena opción para la mejoría de los signos y síntomas orbitales, tumores difusos, afectación de músculos extraoculares, nervio óptico, y afectación ósea, teniendo buenos resultados en la función de la visión con dosis totales de 30-50 Gy ${ }^{18-20}$. En estudios recientes, la radiocirugía estereotáctica se ha implementado en casos de recaída o metástasis refractaria por cáncer tiroideo, y también se han usado inhibidores de la tirosina cinasa que han logrado una reducción de la proliferación celular tumoral, un aumento de la apoptosis y una disminución de angiogénesis ${ }^{19,20}$. Las complicaciones o los riesgos más frecuentes de la cirugía descompresiva o citorreducción secundaria a compresión son la diplopía y el estrabismo, el cual se ha relacionado con trastornos mecánicos ${ }^{16,17,20}$. En nuestra paciente se realizaron estudios preoperatorios complementarios dirigidos a la presentación clínica que mostraba, razón por la cual se realizó una BAAF, que confirmó el cáncer tiroideo variante papilar como origen primario de la lesión orbitaria. Considerando los estudios de imagen sugerentes de una lesión sólida infiltrativa, se consideró la imposibilidad de realizar una resección total por riesgo de distopía del globo ocular. Como el principal síntoma era la neuralgia, se optó por realizar una descompresión del foramen óptico y la fisura supraorbitaria, con buenos resultados, resolviendo los episodios de cefalea y mejorando significativamente la neuralgia. Debido a su presentación clínica y estadio, será intervenida por el servicio de oncocirugía para tiroidectomía total más radioterapia adyuvante, pues en este tipo de lesiones es indispensable el manejo multidisciplinario. Aunque el pronóstico de las metástasis orbitarias es malo, existen numerosos casos de supervivencia prolongada después del tratamiento combinado.

\section{Conclusiones}

La metástasis orbitaria con extensión intracraneal debe ser abordada quirúrgicamente. Las lesiones con difícil acceso para la resección deberán ser valoradas minuciosamente, definiendo la clínica principal, ya que en algunos casos la citorreducción y la descompresión orbitaria mejoran la sintomatología y tiene un impacto positivo en la calidad de vida de los pacientes.

\section{Agradecimientos}

Este trabajo fue previamente revisado y aprobado por todos los autores para su publicación, por lo que se agradece a este equipo de trabajo su tiempo y esfuerzo.

\section{Responsabilidades éticas}

Protección de personas y animales. Los autores declaran que para esta investigación no se han realizado experimentos en seres humanos ni en animales.

Confidencialidad de los datos. Los autores declaran que han seguido los protocolos de su centro de trabajo sobre la publicación de datos de pacientes.

Derecho a la privacidad y consentimiento informado. Los autores han obtenido el consentimiento informado de los pacientes y/o sujetos referidos en el artículo. Este documento obra en poder del autor de correspondencia.

\section{Financiamiento}

Ninguno de los autores recibió apoyo económico o de otro tipo durante el desarrollo de esta investigación. 


\section{Conflicto de intereses}

\section{No existe conflicto de intereses.}

\section{Bibliografía}

1. Globocan 2018. Population fact sheets, Mexico. Disponible en: http://gco. iarc.fr/today/data/factsheets/populations/484-mexico-fact-sheets.pdf.

2. Granados-García M, Gallegos F, Gurrola-Machuca H, Flores-Hernández L, Pacheco-Bravo I, Villavicencio-Queijeiro MA, et al. Guía de manejo del nódulo tiroideo y del cáncer diferenciado de tiroides de la Sociedad Mexicana de Oncología. Gac Mex Oncol. 2018;17(Supl):5-31.

3. Aneiros Fernández J., Chamorro Clara, Nicolae Alina, Preda Ovidiu, Martínez Francisco Javier, Concha Ángel et al. Metástasis orbitaria intracraneal: primera manifestación clínica de cáncer de tiroides. Departamento de Anatomía Patológica y Radiología, Hospital Universitario Virgen de las Nieves, Granada, España; 2015. p. 1-2.

4. Farina E, Monari F, Tallini G, Repaci A, Mazzarotto R, Giunchi F, et al. Unusual thyroid carcinoma metastases: a case series and literature review. Endocr Pathol. 2016:27:55-64.

5. Amemiya $T$, Hayashida H, Dake $Y$. Metastatic orbital tumors in Japan: a review of the literature. Ophthalmic Epidemiol. 2002;9:35-47.

6. Karcioglu ZA, Croxatto JO. Mechanisms of tumor metastasis in the orbit En: Karcioglu ZA, editor. Orbital tumors. Diagnosis and treatment. $2^{\text {nd }}$ ed. New York: Springer; 2005. p. 34-40.

7. Jiménez-Morales ML, Gómez-Garza G, Criales-Cortés JL, Mora-Tiscareño MA Patología orbitaria en la población pediátrica: revisión de hallazgos mediante resonancia magnética. An Radiol Mex. 2015;14:191-208.

8. Shields JA, Shields CL, Scartozzi R. Survey of 1264 patients with orbital tumors and simulating lesions. The 2002 Montgomery Lecture, part 1. Ophthalmology. 2004;111:997-1008.

9. Magliozzi P, Strianese D, Bonavolont P, Ferrara M, Ruggiero P, Carandente R et al. Orbital metastases in Italy. Int J Ophthalmol. 2015;8:1018-23.
10. Bile-Gui LN, Kouadio E, Ohui-Acko EV, Kabas RM, Koui SB, Diambra LMA, et al. Metastatic thyroid carcinoma presented as a large craniofacial mass: case report and CT findings. Radiol Case Rep. 2019;15:128-32.

11. Sheikh AB, Akhtar A, Tariq U, Sheikh AAE, Siddiqui FS, Bukhari MM Skull metastasis extending to the superior sagittal sinus: an unfamiliar presentation of papillary thyroid carcinoma. Cureus. 2018;10:e2738-48.

12. Shen J, Wang S, Zhao X, Shao X, Jiang X, Dai $Y$, et al. Skull metastasis from follicular thyroid carcinoma: report of three cases and review of literature. Int J Clin Exp Pathol. 2015;8:15285-93.

13. Goldberg RA, Rootman J, Cline RA. Tumors metastatic to the orbit: a changing picture. Surv Ophthalmol. 1990;35:1.

14. González F, Lopez Couto C. Orbital metastases. A report of four cases and a review of the literatura. Arch Soc Esp Oftalmol. 2006;81:451-62.

15. Karcioglu ZA, Croxatto JO. Metastatic tumors. En: Karcioglu ZA, editor. Orbital tumors. Diagnosis and treatment. $2^{\text {nd }}$ ed. New York: Springer; 2005. 279-292

16. Lee HS, Yoo H, Lee SH, Gwak HS, Shin SH. Clinical characteristics and follow-up of intracranial metastases from thyroid cancer. Acta Neurochir (Wien). 2015;157:2185-94.

17. Medel R, Vásquez LM. Orbital Surgery. ESASO Course Series. Basel, Karger. Barcelona, España, 2014, pp. 18-33. doi: 10.1159/isbn.978-3318-02606-1.

18. Haugen BR, Alexander EK, Bible KC, Doherty GM, Mandel SJ, Nikiforov YE, et al. 2015 American Thyroid Association Management Guidelines for Adult Patients with Thyroid Nodules and Differentiated Thyroid Cancer: The American Thyroid Association Guidelines Task Force on Thyroid Nodules and Differentiated Thyroid Cancer. Thyroid. 2016;26:1-133.

19. Hong YW, Lin JD, Yu MC, Hsu CC, Lin YS. Outcomes and prognostic factors in thyroid cancer patients with cranial metastases: a retrospective cohort study of 4,683 patients. Int J Surg. 2018;55:182-7.

20. Henriques de Figueiredo B, Godbert $Y$, Soubeyran I, Carrat X, Lagarde $\mathrm{P}$, Cazeau AL, et al. Brain metastases from thyroid carcinoma: a retrospective study of 21 patients. Thyroid. 2014;24:270-6. 